\title{
Substance P Plays a Critical Role in Photic Resetting of the Circadian Pacemaker in the Rat Hypothalamus
}

\author{
Do Young Kim, ${ }^{1}$ Hee-Cheol Kang, ${ }^{1}$ Hyung Cheul Shin, ${ }^{3}$ Kyoung Jin Lee, ${ }^{2}$ Young Wook Yoon, ${ }^{1}$ Hee Chul Han, ${ }^{1}$ \\ Heung Sik Na, ${ }^{1}$ Seung Kil Hong, ${ }^{1}$ and Yang In Kim ${ }^{1}$ \\ ${ }^{1}$ Department of Physiology and Neuroscience Research Institute, Korea University College of Medicine, Seoul, Korea \\ 136-705, ${ }^{2}$ National Creative Research Initiative Center for Neurodynamics and Department of Physics, Korea University, \\ Seoul, Korea 136-701, and 3Department of Physiology, Hallym University College of Medicine, Chunchon, Korea $200-702$
}

Glutamate is considered to be the primary neurotransmitter in the retinohypothalamic tract (RHT), which delivers photic information from the retina to the suprachiasmatic nucleus (SCN), the locus of the mammalian circadian pacemaker. However, substance $\mathrm{P}$ (SP) also has been suggested to play a role in retinohypothalamic transmission. In this study, we sought evidence that SP from the RHT contributes to photic resetting of the circadian pacemaker and further explored the possible interaction of SP with glutamate in this process. In rat hypothalamic slices cut parasagittally, electrical stimulation of the optic nerve in early and late subjective night produced a phase delay $(2.4 \pm 0.5 \mathrm{hr}$; mean \pm SEM) and advance $(2.6 \pm 0.3 \mathrm{hr})$ of the circadian rhythm of SCN neuronal firing activity, respectively. The SP antagonist L-703,606 (10 $\mu \mathrm{M})$ applied to the slices during the nerve stimulation completely blocked the phase shifts.
Likewise, a cocktail of NMDA (2-amino-5-phosphonopentanoic acid, $50 \mu \mathrm{M})$ and non-NMDA (6,7-dinitroquinoxaline-2,3-dione, 10 $\mu \mathrm{M})$ antagonists completely blocked the shifts. Exogenous application of SP $(1 \mu \mathrm{M})$ or glutamate $(100 \mu \mathrm{M})$ to the slices in early subjective night produced a phase delay $(\sim 3 \mathrm{hr})$ of the circadian firing activity rhythm of SCN neurons. Coapplication of the NMDA and non-NMDA antagonist cocktail (as well as L-703,606) resulted in a complete blockade of the SP-induced phase delay, whereas $\mathrm{L}-703,606(10 \mu \mathrm{M})$ had no effect on the glutamate-induced delay. These results suggest that SP, as well as glutamate, has a critical role in photic resetting. Furthermore, the results suggest that the two agonists act in series, SP working upstream of glutamate.

Key words: brain slice; circadian rhythm; electrophysiology; glutamate; hypothalamus; pacemaker; photic resetting; rat; RHT; SCN; substance $P$
The retinohypothalamic tract (RHT) is a neural pathway arising from a subset of retinal ganglion cells and projecting to the suprachiasmatic nucleus (SCN) (Hendrickson et al., 1972; Moore and Lenn, 1972), a hypothalamic region that contains the circadian pacemaker in mammals (Meijer and Rietveld, 1989; Morin, 1994). Photic information conveyed to the SCN via the RHT is sufficient and essential for entrainment of the circadian pacemaker to the environmental light/dark cycle (Rusak and Boulos, 1981). Previous studies have provided strong evidence that the excitatory amino acid (EAA) glutamate is a neurotransmitter used by the RHT (for review, see Ebling, 1996).

However, accumulating data have suggested that substance $\mathrm{P}$ (SP) also plays a neurotransmitter or neuromodulator role in retinohypothalamic transmission. SP-containing nerve fibers are concentrated in the ventral region (i.e., the retinorecipient portion) of the rat SCN (Takatsuji et al., 1991; Mikkelsen and Larsen, 1993), and retinal fibers make synaptic contacts with SP receptor-positive dendrites in the ventral part of the rat SCN (Takatsuji et al., 1995). SP applied exogenously to rodent hypo-

\footnotetext{
Received Dec. 21, 2000; revised March 12, 2001; accepted March 14, 2001.

This work was supported by a grant from the Hallym Academy of Sciences and by Grant 2000-A04+ from Medical Science Research Center at Korea University to Y.I.K. D.Y.K., H.C.H., H.S.N., and Y.I.K. were supported by the Brain Korea 21 Project in 2000. K.J.L. was supported by Creative Research Initiatives of the Korean Ministry of Science and Technology. We are grateful to Dr. C. Allen at Oregon Health Science University for his constructive comments on the previous drafts of this paper.

Correspondence should be addressed to Dr. Yang In Kim, Department of Physiology, Korea University College of Medicine, 126-1 Anam-dong 5-ga, Sungbuk-gu, Seoul, Korea 136-705. E-mail: yikim@korea.ac.kr.

Copyright (C) 2001 Society for Neuroscience 0270-6474/01/214026-06\$15.00/0
}

thalamic slices alters the unit activity of SCN neurons (Shibata et al., 1992; Shirakawa and Moore, 1994; Piggins et al., 1995) and can cause phase shifts in the circadian rhythms of SCN neuronal activity (Shibata et al., 1992). Intracerebroventricular or systemic injection of an SP antagonist in the hamster blocks the expression of Fos-like protein in the SCN (Abe et al., 1996) and the phase advances of circadian locomotor activity rhythm (Challet et al., 1998) induced by light pulses. Moreover, SP antagonist applied to rat hypothalamic slices depresses EPSCs of SCN neurons evoked by optic nerve stimulation (Kim et al., 1999).

On the other hand, there are also some results that throw doubt on the role of SP in retinohypothalamic transmission. Neurons expressing SP receptors in the rat $\mathrm{SCN}$ are mainly located in the dorsolateral margin of the nucleus, not the retinorecipient region (Mick et al., 1994), and the effects of ocular enucleation on the density of SP-immunoreactive nerve fibers and terminals in the SCN are inconsistent; although a few laboratories have reported a significant reduction of SP immunoreactivity in the rat SCN (Takatsuji et al., 1991; Mikkelsen and Larsen, 1993), others have failed to detect a decrease in SP-immunoreactive elements in both the rat and hamster SCN (Otori et al., 1993; Hartwich et al., 1994). Thus, it still remains to be determined whether or not SP plays an RHT neurotransmitter or neuromodulator role.

In this study, using rat hypothalamic slices containing both the SCN and optic nerve, we sought physiological evidence that SP from RHT terminals contributes to photic resetting of the suprachiasmatic circadian pacemaker. In addition, we explored the possible interactions between SP and glutamate in photic resetting. In the first set of experiments, we examined the effects of SP 
and EAA antagonists on the phase shifts of circadian firing rhythms induced by optic nerve stimulation. In the second set, we examined the effects of SP and EAA antagonists on the phaseshifting effect of exogenously applied SP or glutamate.

\section{MATERIALS AND METHODS}

Animals and housing. Male Sprague Dawley rats ( $n=72 ; 50-200 \mathrm{gm})$ purchased from Daehan Experimental Animal Company (Eumsung, Korea) were used in this study. The experimental procedures described below were in accordance with the guidelines set by the Korea University College of Medicine Animal Research Policies Committee. Before electrophysiological experiments, the animals were housed in a temperaturecontrolled room $\left(22-24^{\circ} \mathrm{C}\right)$ with a $12 / 12 \mathrm{hr}$ light/dark schedule for at least a week. Zeitgeber time (ZT) 0:00 hr was defined as the time of lights-on, and ZT 12:00 hr was defined as the time of lights-off in the colony.

Brain slice preparation. The rats were anesthetized with sodium pentobarbitone $(100 \mathrm{mg} / \mathrm{kg}$, i.p.) between ZT 10:30 and 11:30 hr, and the brain was quickly excised and submerged in ice-cold physiological saline (composition in mM, $124 \mathrm{NaCl}, 1.3 \mathrm{MgSO}_{4} \cdot 7 \mathrm{H}_{2} \mathrm{O}, 3 \mathrm{KCl}, 1.25 \mathrm{NaH}_{2} \mathrm{PO}_{4}$, $26 \mathrm{NaHCO}_{3}, 2.4 \mathrm{CaCl}_{2}$, and 10 glucose) supplemented with gentamycin (50 mg/l). After $\sim 1 \mathrm{~min}$ of chilling, the brain was trimmed to a block containing the hypothalamus and optic nerves. With the use of a vibroslicer (World Precision Instruments, Sarasota, FL), two parasagittal slices (400-500 $\mu \mathrm{m}$ thickness), each containing one optic nerve (4-7 $\mathrm{mm}$ ) and an SCN, were cut from the tissue block. During the slicing, the tissue was maintained in ice-cold physiological saline. One of the slices was transferred to a modified Hass-type gas interface recording chamber (Haas et al., 1979) and superfused continuously, at a rate of $0.4 \mathrm{ml} / \mathrm{min}$, with warm physiological saline $\left(35^{\circ} \mathrm{C}\right), \mathrm{pH} 7.4$, aerated with $95 \% \mathrm{O}_{2} / 5 \%$ $\mathrm{CO}_{2}$. Warm air $\left(35^{\circ} \mathrm{C}\right)$ humidified by $95 \% \mathrm{O}_{2} / 5 \% \mathrm{CO}_{2}$ was also continuously blown over the slice to further ensure adequate oxygenation of the cells in the tissue.

Optic nerve stimulation and electrophysiological recording. On the first day in vitro, the optic nerve was stimulated with a suction electrode; constant current pulses of $0.5 \mathrm{~mA}$ intensity $(1.0 \mathrm{msec}$ biphasic square wave; $5 \mathrm{~Hz}$ ) or $0 \mathrm{~mA}$ intensity (in the case of sham stimulation) were applied for $15 \mathrm{~min}$, starting at ZT 14:00 hr (i.e., early subjective night) or 22:00 $\mathrm{hr}$ (i.e., late subjective night). To ensure that the stimulation at 0.5 $\mathrm{mA}$ intensity did not activate SCN neurons directly by current spread, we performed some control experiments using the whole-cell current-clamp technique; the electrodes (tip diameter, $\sim 2 \mu \mathrm{m}$; resistances, $\sim 3 \mathrm{M} \Omega$ ) were filled with a solution, $\mathrm{pH} 7.3$, that was composed of (mM): 140 K-gluconate, $10 \mathrm{HEPES}, 2 \mathrm{MgCl}_{2}, 1 \mathrm{CaCl}_{2}, 11$ EGTA, and $2 \mathrm{~K}_{2} \mathrm{ATP}$. In these experiments, the stimulation intensity was varied from 0.1 to 1.0 $\mathrm{mA}$. In no case ( 10 cells tested in three slices) did the stimulation at 0.5 $\mathrm{mA}$ intensity elicit action potentials directly from SCN neurons, but it clearly evoked EPSPs with a constant latency $(9-15 \mathrm{msec}$; in 5 of 10 cells tested in three slices) (Fig. 1).

Extracellular single-unit recordings were made on the second day in vitro, using glass electrodes (4-5 $\mathrm{M} \Omega$ ) filled with $3 \mathrm{M} \mathrm{NaCl}, \mathrm{pH} 7.4$. Typically 8 units were sampled every hour (range, 5-15 units). The sampling was not restricted to any particular region of the SCN. The single-unit activities were recorded for $1 \mathrm{~min}$ for each cell and grouped into a $2 \mathrm{hr}$ running average with a $1 \mathrm{hr}$ lag to determine the time of peak of circadian firing activity rhythm (Ding et al., 1994). This time point was adopted as the phase reference point of the rhythm.

Drugs. In the first set of experiments (see above), the SP antagonist L-703,606 (0.1-10 $\mu \mathrm{M}$; selective for $\mathrm{NK}_{1}$ receptors; Research Biochemicals, Natick, MA) or a cocktail of the NMDA antagonist 2-amino-5phosphonopentanoic acid (AP-5, $50 \mu \mathrm{M}$; Sigma, St. Louis, MO) and the non-NMDA antagonist 6,7-dinitroquinoxaline-2,3-dione (DNQX, 10 $\mu \mathrm{M}$; Sigma) was bath-applied for $30 \mathrm{~min}$ on the first day in vitro. This application started $10 \mathrm{~min}$ before sham or genuine optic nerve stimulation. In the second set of experiments, SP $(1 \mu \mathrm{M}$; Sigma) or glutamate (100 $\mu \mathrm{M}$; Sigma) was bath-applied for $30 \mathrm{~min}$ on the first day in vitro, starting at ZT 14:00 hr. These agents were applied alone or together with L-703,606 $(10 \mu \mathrm{M})$ or the NMDA and non-NMDA antagonist cocktail (50 $\mu \mathrm{M}$ AP-5 plus $10 \mu \mathrm{M}$ DNQX).

Statistical analyses. Numerical data are expressed as the mean \pm SEM. An ANOVA was used to determine the presence of significant variance among data from different experimental groups, and a Tukey test was performed to see whether there was any significant difference between data from two different experimental groups. $p<0.05$ was considered to be significant.

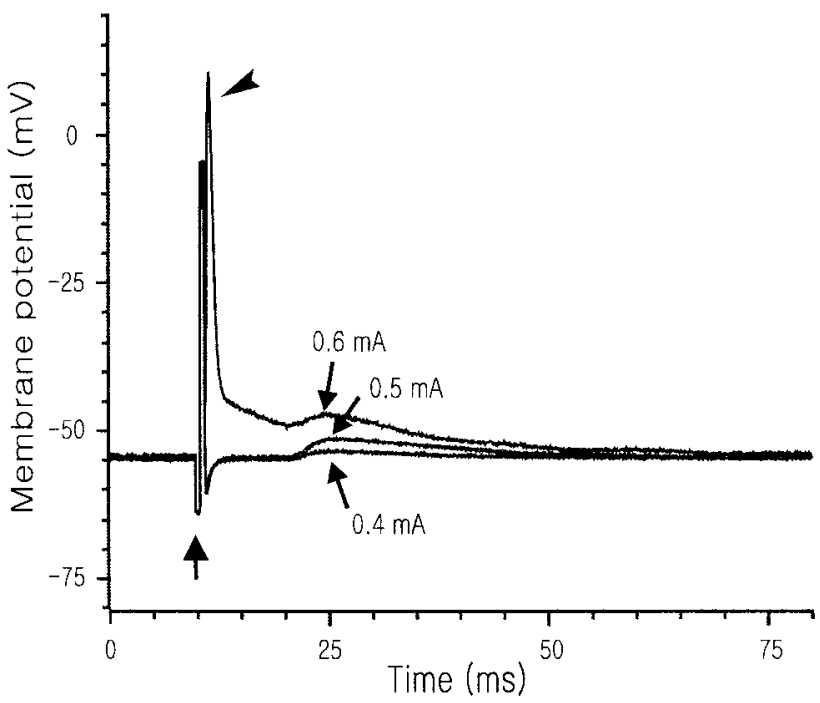

Figure 1. Postsynaptic responses of an SCN neuron to three different intensities of optic nerve stimulation. At all of these intensities, the stimulation (upward arrow) evoked EPSPs with an $11 \mathrm{msec}$ latency. At 0.6 $\mathrm{mA}$ intensity, the stimulation also evoked an action potential (arrowhead) because of current spread to the site of recording; note the short latency of this action potential.

\section{RESULTS}

Effects of SP and EAA antagonists on the phase delays produced by optic nerve stimulation in the early subjective night (ZT $14 \mathrm{hr}$ )

In control slices subjected to sham stimulation of the optic nerve, the peaks of circadian firing activity rhythms were detected at ZT 4-7 hr (ZT $5.8 \pm 0.5 \mathrm{hr} ; n=5$; Fig. $2 A$ ). In optic nervestimulated slices, however, the peaks of circadian rhythms were at ZT 7-10 hr (ZT $8.2 \pm 0.5$ hr; $n=6$; Fig. $2 B$ ), indicating that optic nerve stimulation produced phase delays of the rhythms. The mean delay was $2.4 \pm 0.5 \mathrm{hr}(n=6)$, which was statistically significant (see Fig. 4A).

In slices treated with L-703,606 $(10 \mu \mathrm{M})$ during sham optic nerve stimulation, the peaks of circadian firing activity rhythms were at ZT 5-6 hr (ZT $5.8 \pm 0.3 \mathrm{hr} ; n=4$; Fig. $2 C$ ), similar to those of control slices. This indicated that L-703,606 by itself had no phase-shifting effect. Nevertheless, L-703,606 $(10 \mu \mathrm{M})$ treatment completely blocked the optic nerve stimulation-induced phase delay (Fig. 2D); the times of peaks of circadian rhythms in slices subjected to both optic nerve stimulation and L-703,606 treatment were in the range of ZT 5-6 hr (ZT $5.4 \pm 0.2 \mathrm{hr} ; n=$ $7)$. These peak times were significantly different from those of optic nerve-stimulated, but not control, slices. The effects of 0.1 , 1 , and $10 \mu \mathrm{M} \mathrm{L}-703,606$ on the optic nerve stimulation-induced phase delay are summarized (see Fig. $4 A$ ). Statistically significant blockade of the phase shift was obtained only at $10 \mu \mathrm{M}$, although a partial blockade was noticed at $1 \mu \mathrm{M}$.

Treatment of slices with a cocktail of the EAA antagonists AP-5 $(50 \mu \mathrm{M})$ and DNQX $(10 \mu \mathrm{M})$ was also effective in preventing the optic nerve stimulation-induced phase delay (see Figs. $2 E$, $4 A$ ). In EAA antagonist-treated slices, the times of peaks of circadian rhythms were in the range of ZT 5-7 hr $(\mathrm{ZT} 6.0 \pm 0.3$ hr; $n=5$; Fig. $2 E$ ). Again, these times of peaks were statistically different from those of optic nerve-stimulated, but not control, slices (see Fig. 4A). 

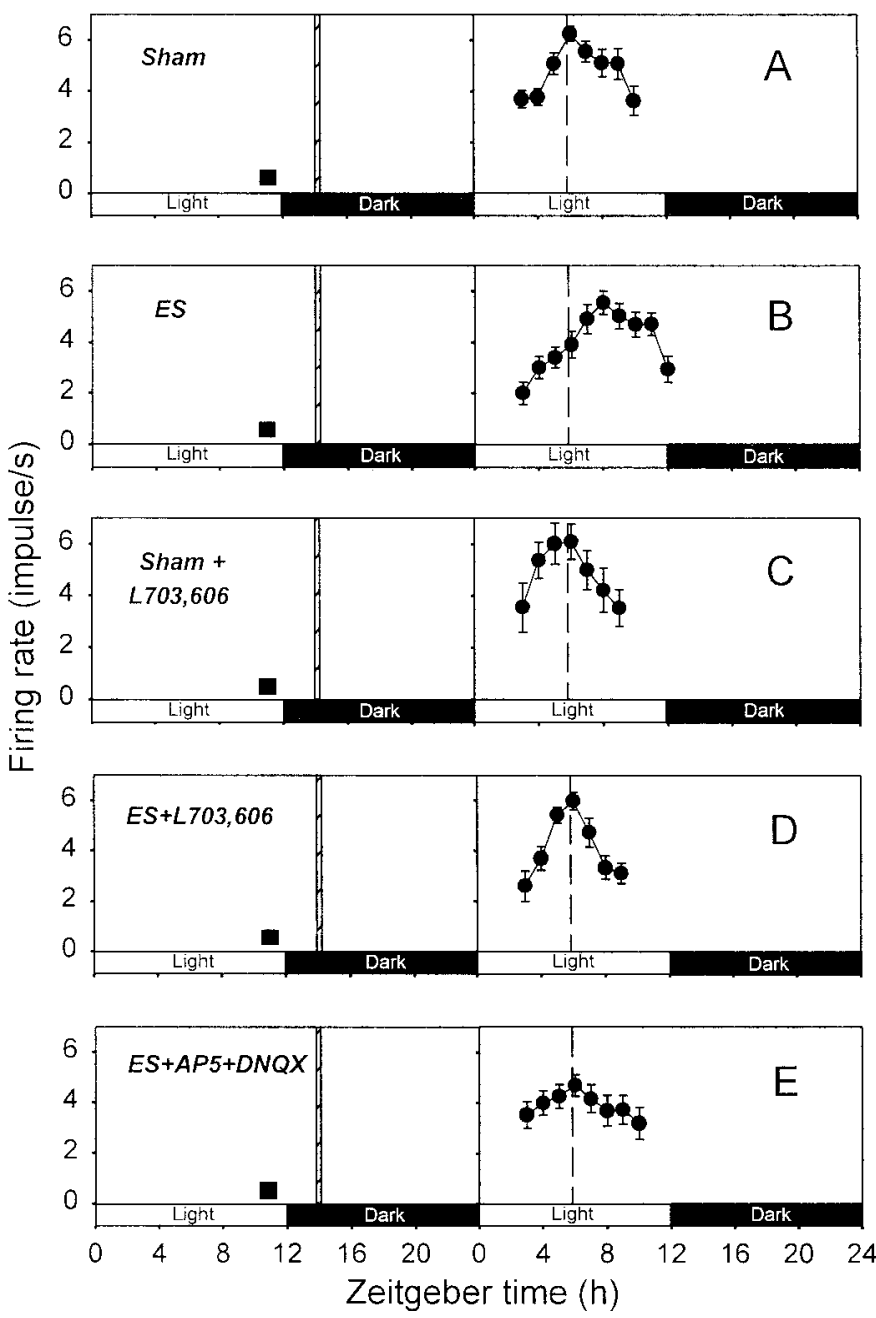

Figure 2. Effects of SP and EAA antagonists on the phase delay of circadian firing rhythm induced by optic nerve stimulation at ZT $14 \mathrm{hr}$. The plots in $A-E$ show representative data from single slices. In each graph, 2 hr running means ( \pm SEM) of firing rates are plotted against $\mathrm{ZT}$ to determine the time of peak firing activity. The projected light and dark phases of the animal room are indicated with open and filled horizontal bars, respectively. Each of the experiments in $A-E$ was repeated four to seven times (see text) for determination of the times of peak firing activities. These were compared across different experiments using ANOVA and Tukey tests. $A$, Sham, Sham stimulation. $B, E S$, Optic nerve stimulation. $C$, Sham $+L 703,606$, L-703,606 application during sham optic nerve stimulation. $D, E S+L 703,606, \mathrm{~L}-703,606$ application during optic nerve stimulation. $E, E S+A P 5+D N Q X$, Application of the AP-5-DNQX cocktail during optic nerve stimulation. Dashed vertical line, Average time of peak firing activity for the slices subjected to sham optic nerve stimulation; filled square, time of slice preparation; hashed vertical bar, time of optic nerve stimulation.

\section{Effects of SP and EAA antagonists on the phase advances produced by optic nerve stimulation in the late subjective night (ZT 22 hr)}

In control slices subjected to sham optic nerve stimulation, the peaks of circadian firing activity rhythms were at ZT 5-6 hr (ZT $5.8 \pm 0.2 \mathrm{hr} ; n=6$; Fig. $3 A$ ). In contrast, in optic nerve-stimulated slices, the peaks were detected at ZT $2-4 \mathrm{hr}$ (ZT $3.2 \pm 0.3 \mathrm{hr} ; n=$ 6; Fig. $3 B$ ), indicating phase advances of the rhythms. These phase shifts $(2.6 \pm 0.3 \mathrm{hr} ; n=6)$ were statistically significant (Fig. $4 B)$.

L-703,606 application $(10 \mu \mathrm{M})$ during sham stimulation of the optic nerve had no significant effect on the phases of circadian
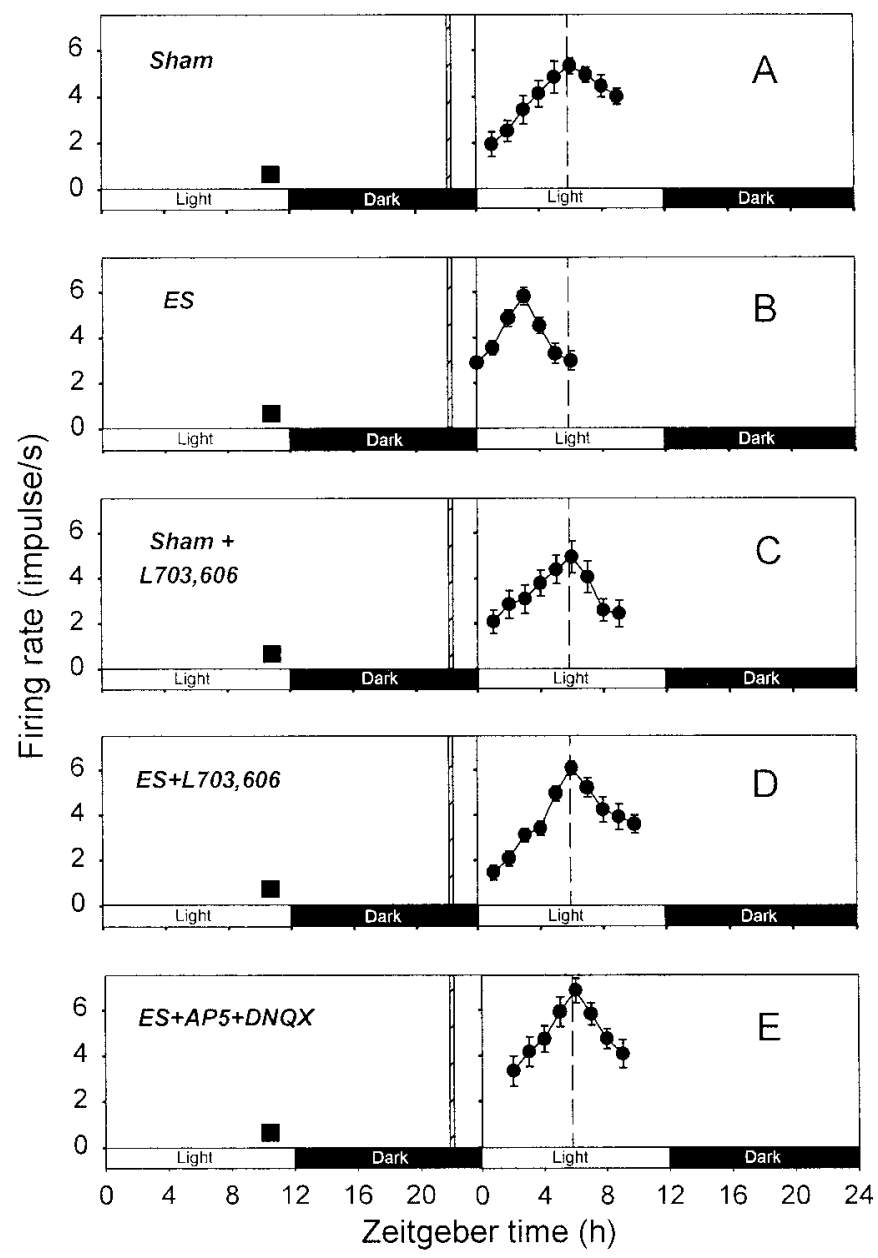

Figure 3. Effects of SP and EAA antagonists on the phase advance of circadian firing rhythm induced by optic nerve stimulation at ZT $22 \mathrm{hr}$. Each of the experiments in $A-E$ was repeated four to six times (see text). The format of this figure is the same as that of Figure 2.

rhythms; their peaks were detected at ZT 5-6 hr $(\mathrm{ZT} 5.5 \pm 0.3$ hr; $n=4$; Fig. 3C). On the other hand, L-703,606 (10 $\mu \mathrm{M})$ treatment completely blocked the phase advance produced by optic nerve stimulation (Figs. 3D, 4B); the times of peaks of circadian rhythms in slices subjected to both optic nerve stimulation and L-703,606 treatment were in the range of ZT 5-7 hr (ZT $6.0 \pm 0.4 \mathrm{hr} ; n=6$ ). These peak times were significantly different from those of optic nerve-stimulated, but not control, slices.

The cocktail of the EAA antagonists was also effective in blocking the optic nerve stimulation-induced phase advance (Figs. $3 E, 4 B$ ). The times of peaks of circadian rhythms in EAA antagonist-treated slices were in the range of $\mathrm{ZT} 5-6 \mathrm{hr}(\mathrm{ZT}$ $5.6 \pm 0.2 \mathrm{hr} ; n=5$ ), which were not statistically different from those of control slices.

\section{Effects of SP and EAA antagonists on the phase delay induced by exogenous SP or glutamate application}

Bath application of SP $(1 \mu \mathrm{M})$ and glutamate $(100 \mu \mathrm{M})$ in the early subjective night produced an average of 3.0 and $3.2 \mathrm{hr}$ of phase delay in circadian firing activity rhythm, respectively (Fig. 5A,D). Coapplication of L-703,606 $(10 \mu \mathrm{M})$ or the NMDA and nonNMDA antagonist cocktail (50 $\mu \mathrm{M}$ AP-5 plus $10 \mu \mathrm{M}$ DNQX) resulted in a complete blockade of the SP-induced phase shift 

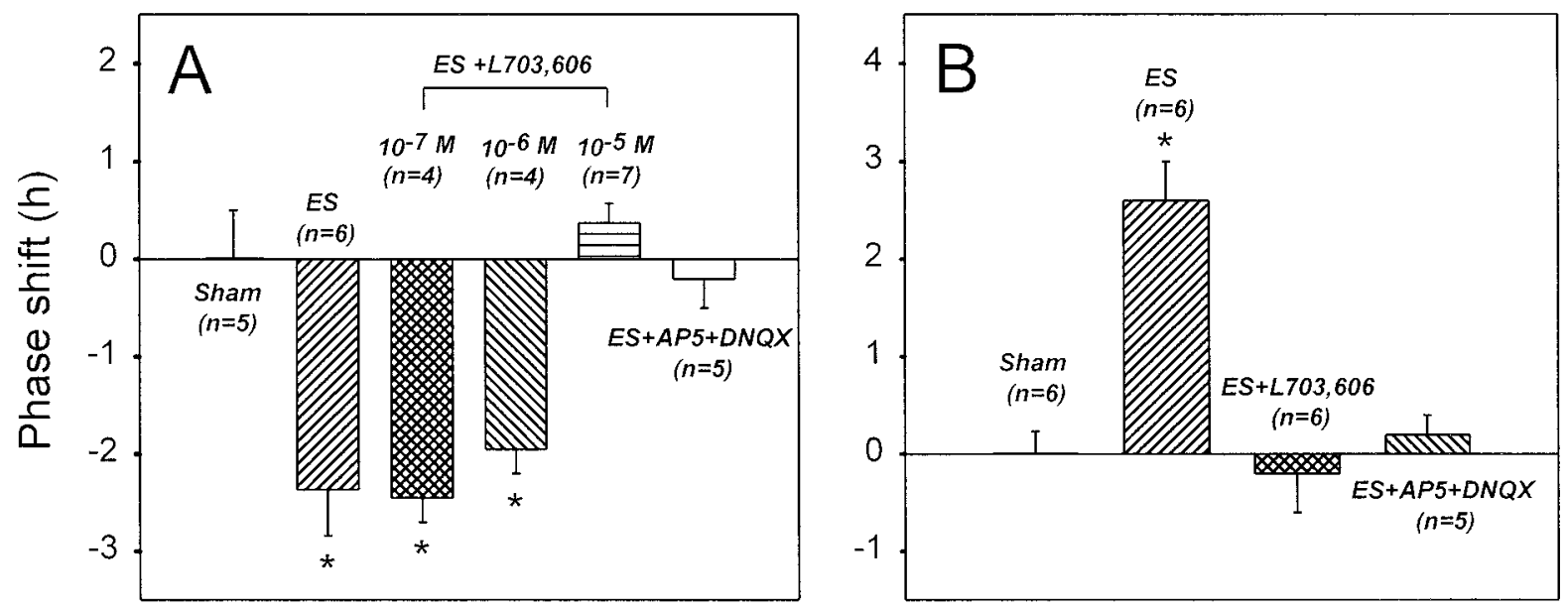

Figure 4. Summary of the effects of SP and EAA antagonists on the optic nerve stimulation-induced phase delay $(A)$ and advance $(B)$. The ANOVA test indicated a significant variance among the data from different experiments $(p<0.001)$ in both $A$ and $B$. The Tukey test further indicated that the asterisk-labeled data were significantly different from the remainder. However, this test indicated that there was no significant difference among the asterisk-labeled data in $A$ and among the remaining data in $A$ and $B$.

(Fig. 5B,C). However, L-703,606 had no significant effect on the glutamate-induced delay (Fig. $5 E$ ).

\section{DISCUSSION}

\section{Role of SP in photic resetting and its interaction with glutamate}

The present study demonstrated that not only the cocktail of NMDA and non-NMDA antagonists but also the selective SP antagonist L-703,606 completely blocked the phase shifts of circadian firing activity rhythm in the SCN induced by optic nerve stimulation. These results strongly suggest that SP, as well as glutamate, released from RHT terminals plays a critical role in photic resetting of the circadian pacemaker. Furthermore, the results suggest that SP and glutamate interact. Previous studies showed that SP potentiated the excitatory responses of SCN neurons to glutamate and NMDA (Shirakawa and Moore, 1994; Piggins et al., 1995). Also, our unpublished data from parasagittal hypothalamic slices indicate that SP potentiates NMDA-induced currents in retinorecipient SCN neurons. Thus, the interaction between SP and glutamate may occur postsynaptically, but it may also occur presynaptically. The finding of Hamada et al. (1999) that SP causes glutamate and aspartate release from hamster SCN slices is consistent with this idea.

SP does not play a permissive role for glutamate and vice versa because glutamate or SP alone was sufficient to produce a phase shift of circadian rhythm (Shibata et al., 1992; Ding et al., 1994). The present study suggests that if the two agonists interact, they act in series with SP working upstream of glutamate, because the SP antagonist L-703,606 had no effect on the glutamate-induced phase shift whereas the EAA antagonist cocktail completely blocked the SP-induced shift. Data from Hamada et al. (1999) showing that the NMDA antagonist MK-801 reduces the SPinduced phase delay of the circadian firing rhythm also provide support for this hypothesis.

The present results suggesting a critical role of SP in photic resetting were somewhat surprising because retinohypothalamic transmission was not dependent on SP (Kim et al., 1999). This study demonstrated that an SP antagonist only slightly depressed the EPSCs of SCN neurons evoked by optic nerve stimulation. However, it is possible that the role of SP was underestimated in this study, because the frequency of optic nerve stimulation was very low $(0.033 \mathrm{~Hz})$. In the present study, the stimulation frequency was $5 \mathrm{~Hz}$. At this frequency, the SP release from optic nerve terminals might be large enough to explain the critical role of SP.

Our observation that the SP antagonist L-703,606 could block both the phase advances and delays of circadian rhythms is not in full agreement with the report of Challet et al. (1998). These investigators found that an intraperitoneal injection of another selective $\mathrm{NK}_{1}$ receptor antagonist in the hamster blocked the light pulse-induced phase advances, not delays, of circadian locomotor activity rhythm. The disparity between our results and those of Challet et al. (1998) might be related to the difference in species; in the rat SCN, SP-containing nerve fibers and terminals are distributed throughout the nucleus, with the largest accumulation in its ventral part (Takatsuji et al., 1991; Mikkelsen and Larsen, 1993), whereas in the hamster SCN, SP-positive fibers are rare although a small cluster of SP neurons are located in the lateral aspect of the nucleus (Morin et al., 1992; Swann and Macchione, 1992; Reuss and Burger, 1994). Thus, in the case of hamster, any SP released from nerve terminals might be derived from intrinsic SCN neurons, rather than from the RHT, and any action of an SP antagonist may be via receptors that never receive input from the RHT.

The disparity between our findings and those of Challet et al. (1998) might also be related to the difference in the site of drug action (SCN vs whole brain) or experimental parameters examined (SCN neuronal firing vs locomotor activity). However, it is unlikely to be caused by nonspecific actions of the SP antagonist used in the present study because (1) L-703,606 had no phaseshifting effects by itself, (2) its effects were concentration dependent, and (3) it produced a selective blockade of the SP-induced (not glutamate-induced) phase delays.

\section{Experimental paradigm}

Recently it was reported that the circadian rhythm of SCN neuronal firing activity, recorded as a single daytime peak in coronal slices of the hamster brain, exhibited two distinct peaks when slices were cut in the horizontal plane (Jagota et al., 2000). In our parasagittal slices of the rat brain, however, dual daytime 

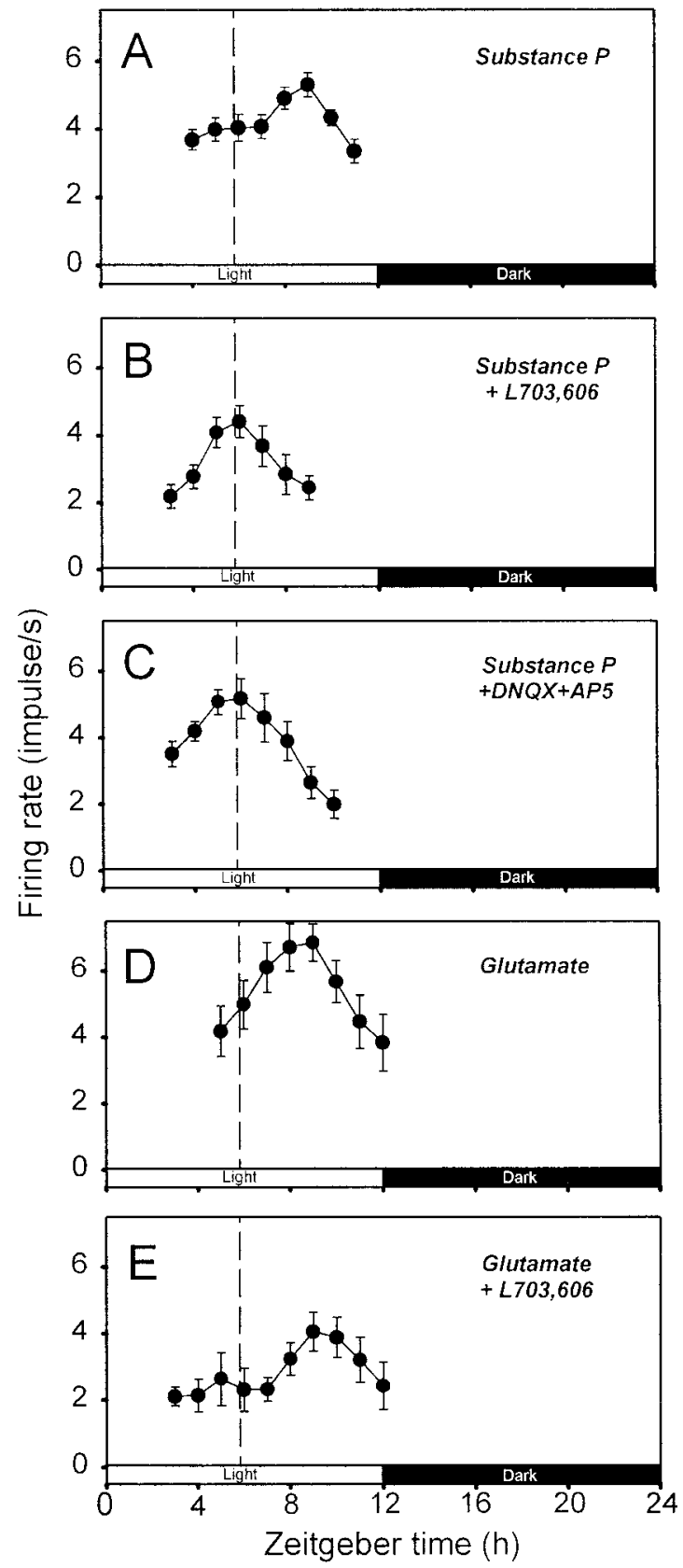

Figure 5. Effects of SP and EAA antagonists on the phase delay induced by exogenous SP or glutamate application. The plots in $A-E$ show representative data from single slices. In each graph, $2 \mathrm{hr}$ running means $( \pm$ SEM) of firing rates are plotted against $\mathrm{ZT}$. The dashed vertical line in each plot indicates the average time of peaks of circadian rhythms observed in control slices (i.e., those subjected to sham optic nerve stimulation). Each of the experiments in $A-E$ was repeated four to six times. The mean times of peaks determined for the experimental conditions in $A-E$ were ZT $8.8 \pm 0.4 \mathrm{hr}(n=6), 5.5 \pm 0.3 \mathrm{hr}(n=4)^{*}, 5.8 \pm$ $0.2 \mathrm{hr}(n=5)^{*}, 9.0 \pm 0.0 \mathrm{hr}(n=4)$, and $9.0 \pm 0.4 \mathrm{hr}(n=4)$, respectively. The ANOVA test indicated a significant variance among these values $(p<0.001)$. The mean values denoted with asterisks in the above list were significantly different from the remainder, but they were not different from each other (Tukey test). The values not labeled with asterisks were not significantly different from one another (Tukey test).

peaks have never been detected. Even in horizontal slices of the rat $\mathrm{SCN}$, only a single peak was detected (P. W. Burgoon, personal communication). Thus, the plane of section is unlikely to be a problem in studying the photic-resetting mechanism.
A potential problem associated with the use of parasagittal slices is the washout of neurotransmitters and neuromodulators in the hours between slice preparation and optic nerve stimulation. However, we had shown that sizable EPSCs, which were sensitive to SP and EAA antagonists, could be evoked in SCN neurons by optic nerve stimulation for up to $12 \mathrm{hr}$ after slice preparation (Kim et al., 1999). Thus, the washout, if any, seems to be a minor problem.

A large number of studies used coronal hypothalamic slices to investigate the postsynaptic mechanisms of photic resetting. In these studies, bath application of a candidate neurotransmitter or neuromodulator of the RHT often substituted for photic stimulation, but direct RHT stimulation was seldom tried (Shibata and Moore, 1993), presumably because of the difficulty of specific RHT stimulation. In the present study, using parasagittal slices, we showed that specific optic nerve (i.e., RHT) stimulation produced a phase advance or delay of the circadian rhythm of SCN neuronal activity with a phase response relationship very similar to that produced by light in vivo. Also, we demonstrated that the phase shifts were sensitive to SP and EAA antagonists. These results suggest that parasagittal hypothalamic slices are a viable in vitro model that allows investigation of both presynaptic and postsynaptic mechanisms of photic resetting.

\section{REFERENCES}

Abe H, Honma S, Shinohara K, Honma K-I (1996) Substance P receptor regulates the photic induction of Fos-like protein in the suprachiasmatic nucleus of Syrian hamsters. Brain Res 708:135-142.

Challet E, Naylor E, Metzger JM, MacIntyre DE, Turek FW (1998) An $\mathrm{NK}_{1}$ receptor antagonist affects the circadian regulation of locomotor activity in golden hamsters. Brain Res 800:32-39.

Ding JM, Chen D, Weber ET, Fairman LE, Rea MA, Gillette MU (1994) Resetting of the biological clock: mediation of nocturnal circadian shifts by glutamate and NO. Science 266:1713-1717.

Ebling FJP (1996) The role of glutamate in the photic regulation of the suprachiasmatic nucleus. Prog Neurobiol 50:109-132.

Haas HL, Schaerer B, Vosmansky M (1979) A simple perfusion chamber for the study of nervous tissue slices in vitro. J Neurosci Methods $1: 323-325$.

Hamada T, Yamanouchi S, Watanabe A, Shibata S, Watanabe S (1999) Involvement of glutamate release in substance P-induced phase delays of suprachiasmatic neuron activity rhythm in vitro. Brain Res 836:190-193.

Hartwich M, Kalsbeek A, Pévet P, Nürnberger F (1994) Effects of illumination and enucleation on substance-P-immunoreactive structures in subcortical visual centers of golden hamster and Wistar rat. Cell Tissue Res 277:351-361.

Hendrickson AE, Wagoner N, Cowan WM (1972) An autoradiographic and electron microscopic study of retino-hypothalamic connections. Z Zellforsch Mikrosk Anat 135:1-26.

Jagota A, de la Iglesia HO, Schwartz WJ (2000) Morning and evening circadian oscillations in the suprachiasmatic nucleus in vitro. Nat Neurosci 3:372-376.

Kim YI, Kim SH, Kim DY, Lee HW, Shin H-C, Chung J-M, Han HC, Na HS, Hong SK (1999) Electrophysiological evidence for the role of substance $\mathrm{P}$ in retinohypothalamic transmission in the rat. Neurosci Lett 274:99-102.

Meijer JH, Rietveld WJ (1989) Neurophysiology of the suprachiasmatic circadian pacemaker in rodents. Physiol Rev 69:671-707.

Mick G, Maeno H, Kiyama H, Tohyama M (1994) Marginal topography of neurons expressing the substance $\mathrm{P}$ receptor in the rat suprachiasmatic nucleus. Brain Res Mol Brain Res 21:157-161.

Mikkelsen JD, Larsen PJ (1993) Substance P in the suprachiasmatic nucleus of the rat: an immunohistochemical and in situ hybridization study. Histochemistry 100:3-16.

Moore RY, Lenn NJ (1972) A retinohypothalamic projection in the rat. J Comp Neurol 146:1-14.

Morin LP (1994) The circadian visual system. Brain Res Rev 67:102-127. 
Morin LP, Blanchard J, Moore RY (1992) Intergeniculate leaflet and suprachiasmatic nucleus organization and connections in the golden hamster. Vis Neurosci 8:219-230.

Otori Y, Tominaga K, Fukuhara C, Yang J, Yamazaki S, Cagampang FR, Okamura H, Inouye ST (1993) Substance P-like immunoreactivity in the suprachiasmatic nucleus of the rat. Brain Res 619:271-277.

Piggins HD, Cutler DJ, Rusak B (1995) Ionophoretically applied substance $\mathrm{P}$ activates hamster suprachiasmatic nucleus neurons. Brain Res Bull 37:475-479.

Reuss S, Burger K (1994) Substance P-like immunoreactivity in the hypothalamic suprachiasmatic nucleus of Phodopus sungorus: relation to daytime, photoperiod, sex and age. Brain Res 638:189-195.

Rusak B, Boulos Z (1981) Pathways for photic entrainment of mammalian circadian rhythms. Photochem Photobiol 34:267-273.

Shibata S, Moore RY (1993) Neuropeptide Y and optic chiasm stimulation affect suprachiasmatic nucleus circadian function in vitro. Brain Res 615:95-100.
Shibata S, Tsuneyoshi A, Hamada T, Tominaga K, Watanabe S (1992) Effect of substance $\mathrm{P}$ on circadian rhythms of firing activity and the 2-deoxyglucose uptake in the rat suprachiasmatic nucleus in vitro. Brain Res 597:257-263.

Shirakawa T, Moore RY (1994) Responses of rat suprachiasmatic nucleus neurons to substance $\mathrm{P}$ and glutamate in vitro. Brain Res 642:213-220.

Swann JM, Macchione N (1992) Photoperiodic regulation of substance P immunoreactivity in the mating behavior pathway of the male golden hamster. Brain Res 590:29-38.

Takatsuji K, Miguel-Hidalgo J-J, Tohyama M (1991) Substance $\mathrm{P}$-immunoreactive innervation from the retina to the suprachiasmatic nucleus in the rat. Brain Res 568:223-229.

Takatsuji K, Senba E, Mantyh PW, Tohyama M (1995) A relationship between substance $P$ receptor and retinal fibers in the rat suprachiasmatic nucleus. Brain Res 698:53-61. 